\title{
The dependence of maximum starspot amplitude and the amplitude distribution on stellar properties
}

\author{
Steven H. Saar ${ }^{1}$, Michelle Dyke ${ }^{1,2}$, Søren Meibom ${ }^{1}$ and \\ Sydney A. Barnes ${ }^{3}$ \\ ${ }^{1}$ Smithsonian Astrophysical Obs., \\ 60 Garden Street, Cambridge,MA 02138, USA \\ email: saar@cfa.harvard.edu, smeibom@cfa.harvard.edu \\ ${ }^{2}$ Yale Univ. New Haven, CT USA \\ ${ }^{3}$ Lowell Obs., 1400 W. Mars Hill Road, Flagstaff, AZ 86011 \\ email: barnes@lowell.edu
}

\begin{abstract}
We combine photometric data from field stars, plus over a dozen open clusters and associations, to explore how the maximum photometric amplitude $\left(A_{\max }\right)$ and the distribution of amplitudes varies with stellar properties. We find a complex variation of $A_{\max }$ with inverse Rossby number $\mathrm{Ro}^{-1}$, which nevertheless can be modeled well with a simple model including an increase in $A_{\max }$ with rotation for low $\mathrm{Ro}^{-1}$, and a maximum level. $A_{\max }$ may then be further affected by differential rotation and a decline at the highest $\mathrm{Ro}^{-1}$. The distribution of $A_{\text {spot }}$ below $A_{\max }$ varies with $\mathrm{Ro}^{-1}$ : it peaks at low $A_{\text {spot }}$ with a long tail towards $A_{\max }$ for low $\mathrm{Ro}^{-1}$, but is more uniformly distributed at higher $\mathrm{Ro}^{-1}$. We investigate further dependences of the $A_{\text {spot }}$ distributions on stellar properties, and speculate on the source of these variations.
\end{abstract}

Keywords. Stars: spots, stars: rotation, stars: magnetic fields, stars: late-type, stars: evolution

\section{Introduction and observations}

A number of extensive photometric studies of open clusters and associations have been completed recently. Since the major compilation and study of spot amplitudes $A_{\text {spot }}$ was by Messina et al. (2001), it timely to revisit the dependence of $A_{\text {spot }}$ (indicative of starspots) on other stellar properties, informed by the new data and other recent results (e.g., on differential rotation). We have collected photometric $\mathrm{V}$ amplitude and rotation period $P_{\text {rot }}$ data from a large number of sources (Table 1) and made new fits to the light curves for two clusters (M35, NGC 3532). The total data set includes over 1200 stars with ages ranging from $10 \mathrm{Myr}$ to roughly solar. We include only single stars or well separated binaries to avoid complications due to tidal/magnetic interactions, and difficulties in assigning amplitudes to a particular component. We also restrict ourselves to $V$ data, which are more numerous (sadly, eliminates some recent cluster surveys).

\section{Analysis and models}

Based on Messina et al. (2001) we expect $A_{\text {spot }}$ to depend on both rotation and mass. A simple, physically motivated parameterization for this is the inverse Rossby number $\mathrm{Ro}^{-1}=\tau_{C} \Omega$ (where $\tau_{C}$ is the convective turnover timescale); the mean-field $\alpha \Omega$ dynamo 
Table 1. Sources of $A_{\text {spot }}$ data

\begin{tabular}{|l|c|c|c|}
\hline Source & $N_{\text {star }}$ & Age $[\mathrm{Myr}]$ & reference \\
\hline $\begin{array}{l}\text { P Pic assoc. } \\
\text { Tuc/Hor assoc. }\end{array}$ & 17 & 10 & Messina et al. $(2010 \mathrm{~b})$ \\
Col assoc. & 52 & 30 & Messina et al. $(2010 \mathrm{~b})$ \\
Car assoc. & & & \\
NGC 2391 & 16 & 40 & Patten \& Simon $(1996)$ \\
NGC 2602 & 29 & 40 & Barnes et al. $(1999)$ \\
IC 4665 & 8 & 40 & Allain et al. $(1996)$ \\
$\alpha$ Per & 42 & 70 & Messina et al. $(2001)^{1}$ \\
Pleiades & 57 & 100 & Messina et al. $(2001)^{1}$ \\
AB Dor group & 32 & 100 & Messina et al. $(2010 \mathrm{~b})$ \\
M35 & 218 & 150 & Meibom et al. $(2009)$ \\
M11 & 8 & 215 & Messina et al. $(2010 \mathrm{a})$ \\
NGC 3532 & 73 & 300 & Barnes $(2003)^{2}$ \\
M37 & 504 & 550 & Hartman et al. $(2009)$ \\
Coma & 39 & 600 & Collier Cameron et al. $(2009)$ \\
Hyades & 21 & 600 & Messina et al. $(2001)^{1}$ \\
field & 99 & $300-4000$ & Messina et al. $(2001)^{1}$ \\
\hline
\end{tabular}

Notes: ${ }^{1}$ Data compilation (see references for original source); ${ }^{2} P_{\text {rot }}$ source, we measure $A_{\text {spot }}$ for the first time.

number is proportional to $\mathrm{Ro}^{-2}$, and $\mathrm{Ro}^{-1}$ figures prominently in stellar activity and rotational evolution (e.g., Barnes \& Kim 2010). We take $\tau_{C}$ from Gunn et al. (1998).

Since $A_{\text {spot }}$ is a relative measure, yielding the peak difference in spot coverage over rotation, we expect that the maximum $A_{\text {spot }}$ seen at a given $\mathrm{Ro}^{-1}, A_{\mathrm{max}}$, will be the most indicative diagnostic of the true strength of dynamo-driven spot generation at that rotation rate. We study $A_{\max }$ (Fig. 1), the binned $A_{\text {spot }}\left(\mathrm{Ro}^{-1}\right)$ distributions, and moments thereof (Fig. 2).

If $A_{\max }$ behaves like other magnetic activity diagnostics (e.g., X-ray emission), we expect to see an increase in $A_{\max }$ with rotation at lower $\mathrm{Ro}^{-1}$, a possible maximal "saturated" spottedness level above some critical $\mathrm{Ro}^{-1}$, and a possible decrease in $A_{\max }$

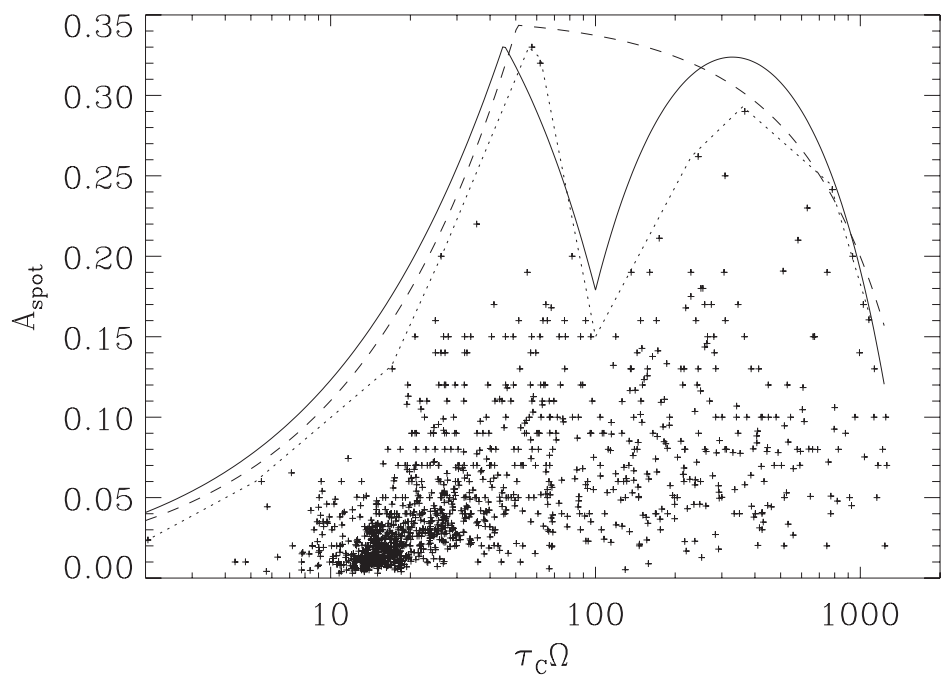

Figure 1. $A_{\text {spot }}$ vs. Ro $^{-1}$ for our sample (Table 1). The dashed line connects local $A_{\text {spot }}$ maxima $\left(A_{\max }\right)$; the dashed and solid lines gives our approximate models ( 1 and 2 , respectively) for $A_{\mathrm{max}}$ (see discussion). 
again at very fast rotation rates (e.g., analogous to the "super-saturation" seen in coronae; e.g., Randich 1998). Causes for the latter phenomena are much debated, and part of the reason for such a study. Saturation may result from maximum surface coverage by fields/spots, Coriolis force-driven concentration of spots at poles, maximum dynamo output limited by back-reaction of magnetic fields on flows. A decline at the highest $\mathrm{Ro}^{-1}$ may be due to a continuation of the above, or simply due to lack of additional surface area to permit higher levels of inhomogeneity.

Connecting the highest observed $A_{\max }\left(\mathrm{Ro}^{-1}\right)$ (Fig. 1, dotted line) does indeed show a general rise in $A_{\max }$ at lower $\mathrm{Ro}^{-1}$, and a decrease at the highest $\mathrm{Ro}^{-1}$. For intermediate values, however, the situation is less clear. One could more coarsely follow $A_{\text {max }}\left(\mathrm{Ro}^{-1}\right)$, and connect $A_{\max }$ high points at $\mathrm{Ro}^{-1} \approx 60$ and 350 , making a fairly smooth, though gently declining "saturated" $A_{\max }$ level. Alternatively, connecting $A_{\max }$ as indicated in Figure 1 shows a large "wedge" removed, with local minimum at $\mathrm{Ro}^{-1} \approx 100$.

We have tried to construct simple models to match both of these scenarios. The first ("no-wedge") $A_{\max }\left(\mathrm{Ro}^{-1}\right)$ curve is well fit by:

$$
A_{\text {max }}=\min \left\{a_{1} \mathrm{Ro}^{-b_{1}} ; c_{1}\left(2-e^{d_{1} / \mathrm{Ro}}\right)\right\}
$$

where $a_{1}, b_{1}, c_{1}$, and $d_{1}$ are adjustable constants. This model contains both a power law increase in $A_{\max }$, limited by a gradually decaying "saturation" level (the exponential term). The $A_{\max }\left(\mathrm{Ro}^{-1}\right)$ curve with the "wedge" removed, can, surprisingly be fit by adding only one additional parameter:

$$
A_{\text {max }}=\min \left\{a_{2} \operatorname{Ro}^{-b_{2}} ; c_{2}\left(2-e^{d_{2} / \mathrm{Ro}}\right)\right\}-f_{2} \Delta \Omega
$$

where $a_{2}, b_{2}, c_{2}, d_{2}$, and $f_{2}$ are adjustable constants, and $\Delta \Omega$ is the empirical relationship between surface differential rotation (DR) and $\mathrm{Ro}^{-1}$ for single dwarfs (Saar 2009; 2010, this volume). The latest fits for this yield $\Delta \Omega \propto \mathrm{Ro}^{-1.00}$ for $\mathrm{Ro}^{-1}<100$ and $\Delta \Omega \propto \mathrm{Ro}^{1.28}$ for $\mathrm{Ro}^{-1}>100$ (Saar 2010, this volume).

We also binned the $A_{\max }$ data into 12 bins of equal numbers in each (100 stars). We also studied the resulting distributions in each bin. Maxima and moments of these distributions are shown in Fig. 2.
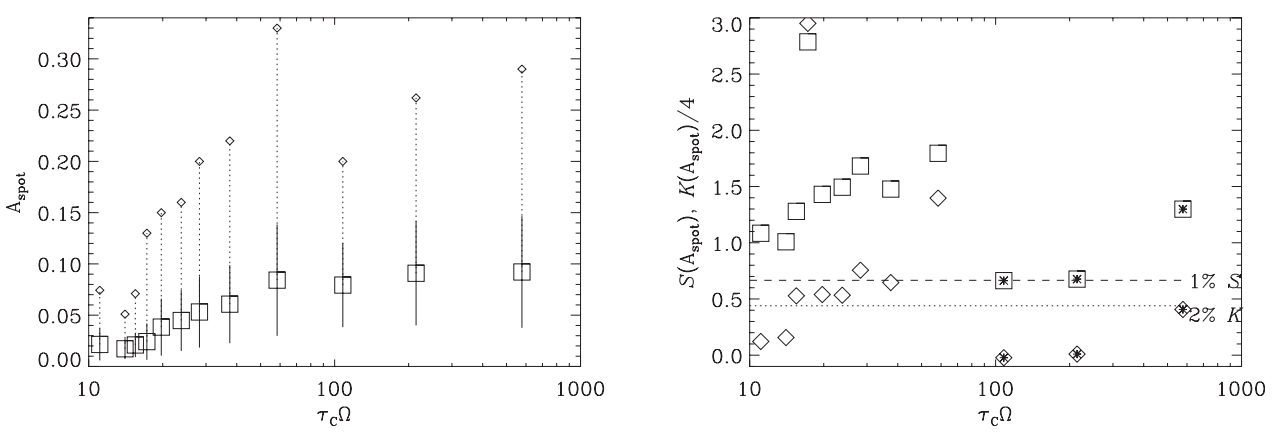

Figure 2. [Left]: The mean (boxes), $\pm \sigma$ width (solid), and maximum (diamonds) of the $A_{\text {spot }}\left(\mathrm{Ro}^{-1}\right)$ distribution in each of 12 bins with 100 stars in each. All three increase fairly steadily until the coronal saturation point $\left(\mathrm{Ro}^{-1} \approx 100\right)$. The mean $A_{\text {spot }}$ also saturates here, while $A_{\max }$ and the distribution $\sigma$ retreat, only to resume increasing towards higher $\mathrm{Ro}^{-1}$. [Right]: Skewness $S$ (boxes) and excess kurtosis $K / 4$ (diamonds) of the $A_{\text {spot }}\left(\mathrm{Ro}^{-1}\right)$ distributions in 100 star bins. Similar to the distribution $\sigma$ width (at left), $S$ and $K$ both increase with $\mathrm{Ro}^{-1}$ below saturation $\left(\mathrm{Ro}^{-1} \approx 100\right)$, whereafter they sharply decline (symbols marked with *). The $1 \%$ significance level for $S$ (dashed) and $2 \%$ level for $K$ (dotted) are also indicated. 


\section{Results and conclusions}

We interpret the success of the added DR term as arising from the effect of strong shear on spot groups, smearing out the spot concentrations (and shredding the spots themselves), inevitably leading to more uniform spatial distributions and lower $A_{\max }$. The shearing effect of DR reaches a peak in a range of rapid rotators near the start of the "saturated activity" branch (where, e.g., $L_{X} / L_{\mathrm{bol}}$ reaches a constant maximum of $\approx 10^{-3}$ ). The sharp increase in $A_{\max }$ for $\mathrm{Ro}^{-1}>100$ (Fig. 1) echoes the Messina et al. (2001) result, which we suggest is due to decreasing rotational shear in these stars, permitting larger spots, spot groups, and thence larger $A_{\max }$.

There are also a significant changes in $A_{\text {spot }}$ distribution around $\mathrm{Ro}^{-1} \sim 100$ : it becomes narrower (Fig. 2, left), and markedly less skewed and more Gaussian (excess kurtosis $K \rightarrow 0$; Fig. 2, right) even while $\left\langle A_{\text {spot }}\right\rangle$ stays roughly constant (Fig. 2, left). These changes, occurring precisely at the local $A_{\max }$ minimum, lends weight to the "wedged" interpretation of $A_{\max }$. Again, high shear should tend to smear out spot groups and structures, making distributions more homogeneous and reducing their moments.

Modeling and further observations are needed to help understand the physical processes underlying these results. Stronger Coriolis forces at high $\mathrm{Ro}^{-1}$ will drive increased flux emergence towards the poles (e.g., Schüssler \& Solanki 1992), but spots are still observed to appear at all latitudes (e.g., Strassmeier 2009), suggesting a source of flux/spots nearer the surface. The implied increase in importance of a convection zone-based dynamo at higher $\mathrm{Ro}^{-1}$ (vs. a tachocline-driven one) is probably an important factor driving changing spot distributions, as are altered velocity fields (e.g., DR, meridional flows). Is there any significance to the sharp spike in $S$ and $K$ near $\mathrm{Ro}^{-1} \approx 17$ ? Is there any relationship between coronal "super-saturation" and the drop in $A_{\max }$ at high $\mathrm{Ro}^{-1}$ ? Do true spot areas continue increasing, even as $\left\langle A_{\text {spot }}\right\rangle$ saturates and $A_{\text {max }}$ declines? Indeed, does $A_{\text {max }}$ decline sharply? (The drop is not apparent in the binned data; Fig. 2, left.) More $A_{\text {spot }}$ data at high $\mathrm{Ro}^{-1}$, and measurements of absolute spot coverage using molecular bands (e.g., ONeal et al. 1996; Saar et al. 2001) could be used to probe these issues. We are also beginning to explore some of these questions with simple models.

\section{Acknowledgement}

This work was supported by Chandra grants GO8-9025A and GO0-11041A.

\section{References}

Allain, S., Bouvier, J., Prosser, C., Marschall, L. A., \& Laaksonen, B. D. 1996, A\&A, 305, 498 Barnes, S. A. \& Kim, Y.-C. 2010, ApJ, 721, 675

Barnes, S. A. 2003, ApJ, 586, 464

Barnes, S. A., Sofia, S., Prosser, C. F., \& Stauffer, J. R. 1999, ApJ, 516, 263

Collier Cameron, A., et al. 2009, MNRAS, 400, 451

Gunn, A. G., Mitrou, C. K., \& Doyle, J. G. 1998, MNRAS, 296, 150

Hartman, J. D., et al. 2009, ApJ, 691, 342

Meibom, S., Mathieu, R. D., \& Stassun, K. G. 2009, ApJ, 695, 679

Messina, S., Desidera, S., Turatto, M., Lanzafame, A. C., \& Guinan, E. F. 2010, arXiv:1004.1959

Messina, S., Parihar, P., Koo, J.-R., Kim, S.-L., Rey, S.-C., \& Lee, C.-U. 2010, A\& A, 513, A29

Messina, S., Rodonò, M., \& Guinan, E. F. 2001, A\&SA, 366, 215

O'Neal, D., Saar, S. H., \& Neff, J. E. 1996, ApJ, 463, 766

Patten, B. M. \& Simon, T. 1996, ApJS, 106, 489 
Randich, S. 1998, 10th Cool Stars, Stellar Systems, and the Sun, ASP Conf. Ser., 154, 501

Saar, S. H. 2009, Astronomical Society of the Pacific Conference Series, ASP Conf. Ser.416, 375

Saar, S. H., Peterchev, A., O’Neal, D., \& Neff, J. E. 2001, 11th Cambridge Workshop on Cool Stars, Stellar Systems and the Sun, ASP Conf. Ser., 223, 1057

Schüssler, M. \& Solanki, S. K. 1992, A\&A, 264, L13

Strassmeier, K. G. 2009, A\&A Rev., 17, 251 